\title{
Regional collaborative forecast of primary energy consumption in China, Japan and South Korea based on multi-source data combination
}

\author{
Bingchun Liu', Peng Zhang ${ }^{1}$, Qingshan Wang ${ }^{2 *}$ \\ 1. Research Institute of Circular Economy, Tianjin University of Technology, Tianjin 300384, China;tjutlbc@tjut.edu.cn (B.L.); \\ tjutfcc@sina.com (C.F.) \\ 2. Tianjin Agricultural University, Tianjin 300384, tigermountain@yeah.net \\ * Correspondence: tjauwangqs@163.com; Tel.: +1-203-392-5763
}

\begin{abstract}
This study aims at improving the forecast accuracy of primary energy consumptions in China, Japan and South Korea and verifying the correlation in primary energy consumptions among the neighboring countries. Considering the diversity of primary energy composition, this study selects 6 components of primary energy, including oil, coal, natural gas, nuclear energy, hydropower and renewable energy as characteristic variables. A collaborative prediction model based on SVR for primary energy consumption prediction is proposed to explore the correlation of primary energy consumption among three countries in China, Japan and South Korea. The results show that there is a strong correlation between primary energy consumption when multiple countries make collaborative prediction, among which the primary energy consumption of South Korea has the largest impact on the primary energy consumption of China and Japan. In the primary energy cooperation of China-Japan-South Korea, a primary energy cooperation system with the South Korea as the link should be established through regional coordination to alleviate the shortage of traditional fossil energy.
\end{abstract}

KEY WORDS Collaborative forecast; Support vector regression; Support vector regression;

China-Japan-South Korea; Primary energy consumption

\section{INTRODUCTION}

Regional cooperation in primary energy consumption is another collaborative approach to resolve unequal resources distribution besides international energy cooperation. As primary energy plays an important role in economic development, countries need to timely adjust energy consumption forms to cope with market fluctuations [1]. Energy strategy [2], energy reserve [3], productivity [4] and geopolitics [3] are crucial for each country engaging in comprehensive energy cooperation. In particular, for primary energy with different strategic status and commodity characteristics, the consumption in different regions is also different, that is, a primary energy consumption directly reflects its strategic status. For example, in East Asia, China, Japan and South Korea have become the world's largest region in terms of primary energy consumption [5]. When considering energy partners, the three countries tend to choose the countries with good economic and political relations in the crude oil market to ensure the reliability and safety of cooperation, while they tend to pay more attention to price and transportation costs. All these considerations will be reflected in the local collaborative patterns. Driven by these factors, energy cooperation in primary energy consumption in China, Japan and South Korea will evolve towards global and regional collaborative pattern. Therefore, studying the correlation pattern of interregional primary energy consumption is a necessary condition for further formulating cooperation strategies between 
countries in the energy market [6]. To understand the correlation of primary energy consumption between different countries can provide targeted suggestions for alleviating the shortage of fossil fuels.

When it comes to the prediction of primary energy consumption, regional collaborative forecast has been rarely used for analyzing regional correlations. For example, Giray Gozgor(2018), for example, took the composition data of primary energy consumption of 29 OECD(Organization of Economic Cooperation and Development) countries from 1990 to 2013 as panel data to compare and analyze the impact of renewable energy and non-renewable energy consumption on economic growth [7]. T. Chen (2015) established a collaborative fuzzy neural network in his study, and combined different models to construct new models to effectively analyze global carbon dioxide concentration [8].However, these researchers lack of targeted exploration of the internal causes and estimation of the future when conducting collaborative research. The regional collaborative prediction used in this paper is based on the combination of multi-source data and provides a new research method to analyze the interaction between regions. It pays special attention to the relationship between regions, predicts primary energy consumption of each country through different data combination forms, and judges the correlation between primary energy consumption of different combination forms [9].

This study analyzes the internal correlation between primary energy consumptions of China, Japan and South Korea with focusing on the collaborative mode and mutual dependence between the three countries, and contributes to collaborative development of primary energy of the three countries. In this paper, machine learning method is used to establish a support vector regression (SVR) regional joint model to predict the primary energy consumption of China, Japan and South Korea [10].Starting from the input variables with consideration of the degree of correlation of primary energy consumptions between the three countries, the study combines the primary energy consumption data in different forms as input variables to predict, and compares the prediction errors of these different combinations. In addition to further improving the prediction accuracy, this study observes the interaction of primary energy between different countries, and analyzes the interaction between regions. Combining the predicted results, it provides targeted policy recommendations for the coordinated development of primary energy among regions.

\section{LITERATURE REVIEW}

\section{A. Primary energy consumption forecast}

In recent years, scholars around the world have studied national and regional primary energy consumption predictions. The research is mainly divided into two categories. On one hand, it uses different characteristic variables to improve the prediction accuracy of primary energy consumption. Gokhan, A (2015) employed economic indicators and demographic statistics as eigenvalues when predicting the primary energy consumption of Turkey [40]. Chen, G.Q.(2015) used production, consumption and international trade as input variables to analyze the global variation trend of primary energy consumption, and further studied the correlations and interactions between global primary energy consumption in production, consumption and international trade[15]. Vincenzo Bianco (2015) compared the influence of different fuels and carbon prices and analyzed the primary energy consumption of Italy's thermal power sector and optimized the proportion of different fuels while meeting primary energy demand.[16]. Akpinar, M.(2016) divided the natural gas consumption of cities into seasonal categories, which is used as a forecasting characteristic index to predict natural gas demand[42]. Brinda Mahalingam (2018) analyzed data on US gross domestic product (GDP) and energy consumption, and proposed the correlation between energy consumption and GDP [13]. Li JR (2018) took GDP, population, export and import data as inputs for the prediction of the total petroleum consumption of China [18]. On the other hand, building hybrid model is to increase the prediction accuracy of primary energy. Torrini, F.C. (2016) predicted Brazil's energy consumption by using fuzzy forecast [39]. Chaoqing Yuan (2016) combined ARIMA model and $\operatorname{GM}(1,1)$ gray model to forecast the primary energy consumption of China, resulting in higher prediction accuracy [17]. SasanBarak (2016) built the ARIMAANFIS hybrid model to forecast the annual energy consumption of Iran [19]. Shaikh, F. (2017) optimized the non-linear gray prediction model and increased the prediction accuracy of natural gas consumption of China [41]. Jin Xiao (2018) established a hybrid prediction model based on data processing selection method to predict the primary energy consumption of China [11]. QiangWang (2018) employed single 
linear, mixed linear and non-linear time series prediction technique to predict the energy demands of China and India [12]. Luisa F.Cabeza (2018) analyzed the global and regional primary data of final energy consumptions in China and India and compared current and historical energy consumption trends through researching on the supply and demand of curve changes [14].

\section{B. COLLABORATIVE FORECAST}

On the prediction study of the problem, researchers will use the method of collaborative prediction to improve the prediction accuracy and explore the correlation between eigenvalues [37]. Toly Chen (2013) proposed an effective fuzzy collaborative prediction method, which used each round of fuzzy artificial neural network training to generate the upper and lower bounds of the operating cycle time, and effectively predicted the working cycle time through the cooperation between the upper and lower bounds[20]. UsamaAl-mulali (2014) used the combination of six eigenvalues to predict carbon dioxide emissions [24]. Can Eksoz(2014) proposed a conceptual framework with aiming at the related factors of collaborative prediction of food supply chain, which improving the long-term and accurate collaborative prediction accuracy of seasonal, perishable, promotional and newly launched products between manufacturers and retailers through cooperative prediction between manufacturers and retailers [21] T.Chen (2015) established a cooperative fuzzy neural network in his study, combined different models to construct a new model to effectively analyze and predict global carbon dioxide concentration [22].Sayyed Mahdi Ziaei (2015) established a grey prediction model, and carbon dioxide emissions of European, east Asian and oceanian countries were used as input variables to analyze their impact on energy consumption and financial indicators [23]. Yanling Liu (2018) combined several prediction methods together for the prediction of natural gas consumption of China [38]. Giray Gozgor (2018) used the data on primary energy consumption composition of 29 member countries in the Organization of Economic Cooperation and Development (OECD) from 1990 to 2013 as the panel data to collaboratively analyze the influence of renewable and non-renewable energy consumptions on economic growth, and predicted the future trend of renewable energy consumption [25]. Mert Topcu (2018) adopted a group framework to discuss the inhomogeneity and trans-sectoral dependence between trade and energy consumption, so as to investigate the trade energy consumption relationship of OECD countries during 2015 [26].

\section{SUPPORT VECTOR REGRESSION}

Support vector machine (SVM) is a rigorous supervised learning model that can classify and regress. This model can introduce nonlinear classifiers for processing various types of input data. Based on SVM, Smola and Vapnik propose regression techniques for support vector regression [27]. It is suitable for prediction and less affected by dimensions. SVR-based engineering applications have been widely applied to the prediction on non-linear data. Yongbao Chen (2015) used SVR to calculate and predict short-term power load and then to calculate the power demand of office buildings [33]. YouLong Yang (2016) described the incremental model for SVR, which optimized the prediction of power load [32]. Andrés García-Floriano (2018) built the SVR model, predicted the software enhancement work and formulated the software enhancement plan [28]. Wentao Yang (2018) established the temporal-spatial SVR model to predict the hourly pm2.5 concentration, and solve spatial heterogeneity [29]. Petra Vrablecová (2018) predicted short-term power load by using SVR based on on-line processing of massive data generated by smart grid, and maximally reduced grid imbalance [30]. Gelayol Golkarnarenji (2018) developed an intelligent prediction model for the carbon fiber industry based on support vector regression (SVR) prediction model and genetic algorithm (GA) to reduce energy consumption [31]. In financial field, SVR is also widely used in data analysis and prediction. Abdolreza Nazemi (2018) applied multi-factor SVR model for the analysis of corporate bonds, predicted corporate bond recovery rate [34]. A.Khosravi (2018) combined neuro-fuzzy inference system, feedforward neural network and SVR model to predict the wind direction and velocity of Bushehr. In terms of prediction of regression models, many researchers have proposed unique regression models to explore the relationship between dependent variables and independent variables according to different research objects and fields[35].

\section{III.SUPPORT VECTOR REGRESSION MODEL}

SVR is built upon statistical learning and emphasizes minimization of structural risk. This technique can overcome the defects of conventional statistical pattern recognition 
methods, including neural network. SVR model derives an approximation function $\mathrm{g}(\chi)$ from miscellaneous data sample. $\mathrm{G}=\left(\mathcal{X}_{i}, \mathcal{Y}_{i}\right)_{(i=1)}^{N}$ SVR model is defined as follows:

$$
\mathrm{y}=\sum_{i=1}^{n} \omega_{i} \cdot \varphi\left(\chi_{\mathrm{i}}\right)+\mathrm{b}
$$

Where $\omega$ is weight vector; $\varepsilon$ is error; $\varphi$ is the mapping function; $b$ is deviation. Coefficient $\omega_{i}$ can be estimated by the minimum function:

$R(w)=\frac{1}{N} \sum_{i=1}^{N}\left|f\left(x_{i}\right)-y_{i}\right|_{\varepsilon}+\lambda w^{2}$

Where $\lambda$ is a standardized constant; function $\left|f\left(x_{i}\right)-y_{i}\right|_{\varepsilon}$ is defined as follows:

$\left|f\left(x_{i}\right)-y_{i}\right|_{\varepsilon}=\left\{\begin{array}{c}|f(x)-y|-\varepsilon,\left|f\left(x_{i}\right)-y_{i}\right| \geqq \varepsilon \\ 0, \quad \text { other }\end{array}\right.$

In addition to formula (2), minimum function can be also expressed in the following form [36]:

$f\left(x, \alpha, \alpha^{*}\right)=\sum_{i=1}^{N}\left(\alpha_{i}-\alpha_{i}^{*}\right) k\left(x_{i}, x\right)+b$

Here, $\quad \alpha \alpha^{*}=0, \alpha, \alpha^{*} \geqq 0, i=1, \ldots, N$ Moreover, kernel function defines the inner product of $D_{i}$-dimensional feature space:

$k(x, y)=\sum_{j=1}^{N} \Phi_{i}(x) \Phi_{j}(y)$

Coefficients ${ }^{\alpha_{i}} \alpha_{i}$ and $\alpha_{i}^{*}$ are given by

$R\left(\alpha_{i}^{*}, \alpha_{i}\right)=-\varepsilon \sum_{i=1}^{N}\left(\alpha_{i}^{*}+\alpha_{i}\right)+\sum_{i=1}^{N} y_{i}\left(\alpha_{i}^{*}-\alpha_{i}\right)-\frac{1}{2} \sum_{i, j=1}^{N}\left(\alpha_{i}^{*}+\alpha_{i}\right)$

$\left(\alpha_{i}^{*}-\alpha_{i}\right) k\left(x_{i}, x_{j}\right)$

The constraint conditions are

$\sum_{i=1}^{N}\left(\alpha_{i}^{*}-\alpha_{i}\right)=\mathbf{0}, \quad \alpha_{i} \geqq \mathbf{0}, \quad \alpha_{i}^{*} \leqq C$

In the present study, programming language is used to realize SVR and the continuous value of primary energy consumption is predicted.

\section{DATA AND RESULTS ANALYSIS}

\section{A. Data Sources}

Due to the Geographical similarity, the primary energy consumption of China, Japan and South Korea from 1965 to 2017 is chosen as the research object. The data sources include total annual primary energy consumptions and annual consumptions of the six primary energy sources respectively (namely, petroleum, coal, natural gas, nuclear energy, hydropower and renewable energy) for the three countries. All these data are referred from the Statistical Yearbooks of British Petroleum Company.

The data of total primary energy consumption and its components in these three countries from 1965 to 2009 were divided into training samples, 45 in total. The primary energy consumptions from 2010 to 2017 were the testing samples, 8 in total (TABLE I).

TABLE I

SAMPLES OF PRIMARY ENERGY CONSUMPTION

\begin{tabular}{|c|c|c|c|}
\hline \multicolumn{2}{|c|}{$\begin{array}{c}\text { Primary energy } \\
\text { consumption }\end{array}$} & \multirow{2}{*}{$\begin{array}{c}\text { Size } \\
1965-2009\end{array}$} & \multirow{2}{*}{$\begin{array}{r}\text { Date } \\
45\end{array}$} \\
\hline China & Training data & & \\
\hline & Testing data & $2010-2017$ & 8 \\
\hline \multirow[t]{2}{*}{ Japan } & Training data & $1965-2009$ & 45 \\
\hline & Testing data & $2010-2017$ & 8 \\
\hline \multirow{2}{*}{$\begin{array}{l}\text { South } \\
\text { Korea }\end{array}$} & Training data & $1965-2009$ & 45 \\
\hline & Testing data & $2010-2017$ & 8 \\
\hline
\end{tabular}

\section{B. Multi-source data collaboration SVR model}

Multi-source data collaboration approach is used for the forecast, which is divided into five steps. Firstly, the consumptions of each of the six primary energy sources for the target country are the input variables of the prediction model, and the total primary energy consumption of this country is the output variables. Then SVR is applied to predict. Secondly, the consumptions of each of the six primary energy sources for the target country plus those of another country (input country 1) are taken as the input variables, 12 in total; the total primary energy consumption of the target country is the output variable. Then SVR is also applied to predict. Thirdly, the consumptions of each of the six primary energy sources for the target country plus those of the last country (input country 2) are taken as the input variables, 12 in total; the total primary energy consumption of the target country is the output variable which is done by SVR. Fourthly, the consumptions of each of the six primary energy sources for the above three countries are taken as the input variables, 18 in total; the total primary energy consumption of the target country is the output variable which is still done by SVR. Finally, the forecast values of primary energy consumption of various combination schemes are compared, and the optimized values will be selected for testing. The experimental steps are illustrated in Fig. 1. 


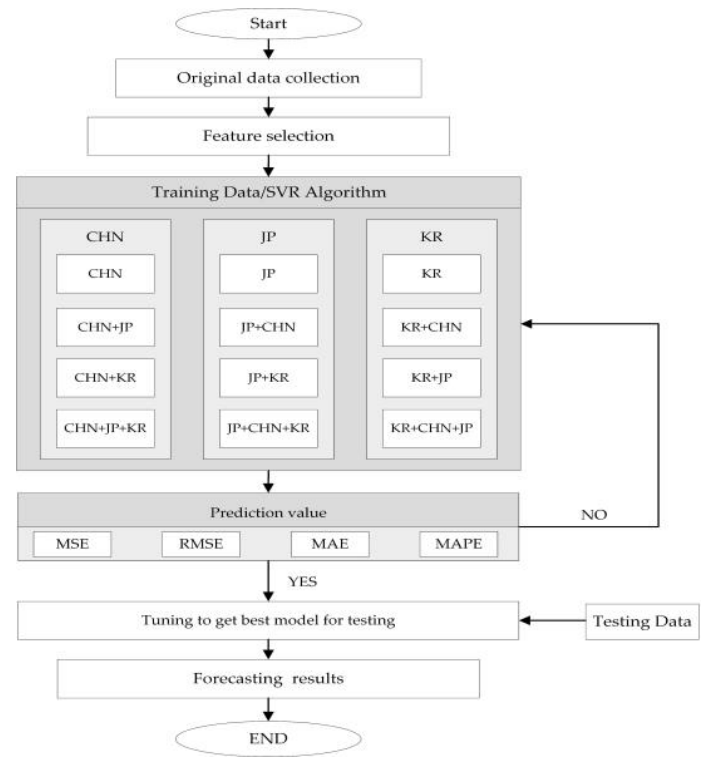

FIGURE 1. Multi-source data collaborative support vector regression model

Prediction errors of the four combinations are calculated by mean square error (MSE), root mean square error (RMSE), mean absolute error (MAE) and mean absolute percentage error (MAPE). The performance of different models is assessed based on these errors. MAPE is the primary error; RMSE, MAE and MAPE are secondary errors for verification the accuracy of MAPE. The MSE, RMSE, MAE and MAPE are defined as:

$$
\begin{aligned}
& M S E=\frac{1}{n} \sum_{i=1}^{n}\left(w_{\text {svr }}-w_{\text {real }}\right)^{2} \\
& M A E=\frac{1}{n} \sum_{i=1}^{n}\left|w_{\text {svr }}-w_{\text {real }}\right| \\
& M S E=\frac{1}{n} \sum_{i=1}^{n}\left(w_{\text {svr }}-w_{\text {real }}\right)^{2} \\
& M A P E=\frac{1}{n} \sum_{i=1}^{n}\left|\frac{w_{\text {svr }}-w_{\text {real }} \mid}{w_{\text {svr }}}\right|
\end{aligned}
$$

where $\omega_{\text {svr }}$ is the total primary energy consumption predicted by the model, and $\omega_{\text {real }}$ is the actual total primary energy consumption. RMSE and MAE are scale dependent measures based on squared and absolute error values. MSE and MAE are less sensitive to outliers, as they accept absolute values without square of the error, but they are easy to calculate. MAE is one of the most common metrics used to calculate error and for comparing different methods on the same datasets. MAPE is based on percentage error and is an independent metric. Therefore, MAPE is the best method for comparing the predictive performance of SVR model on different datasets.

\section{C.ANAL YSIS OF RESULTS}

In this study, multi-source data is used for combination forecasting; therefore a unified performance measurement standard is needed to measure the prediction accuracy of different combinations. MAPE solves this problem. The predictive results vary when using different combinations of primary energy consumption data. When there is a strong correlation between input indicator and output indicator, MAPE will be low.

According to the above design steps, the total annual primary energy consumptions of China are predicted based on multi-source data collaborative forecasting method, which is used for experiments on four combination schemes, namely, China, China+Japan, China+South Korea, and China+South Korea+Japan. The error indicators are MSE, RMSE, MAE and MAPE. The errors of the four combinations for the prediction of the total annual primary energy consumption of China (of four schemes) are shown in Table 2. It can be seen that when using the combination of China+South Korea, the errors are the smallest. That is to say, when the primary energy consumption forecasting of China implements the coordinated forecasting of the secondary energy consumption combination of China+Korea, the improvement effect is very obvious. For the given time span (1965 to 2017), the comparison of the predictive values and actual values of the total primary energy consumption of China by using the four combinations is shown in Fig. 2.

$$
\text { TABLE II. }
$$

China's primary energy consumption forecast is based on the comparison of results of primary energy composition in different countries

\begin{tabular}{cccccc}
\hline \hline Primary energy combination & MSE & \multirow{2}{*}{ RMSE } & MAE & MAPE \\
\hline China & 12390.146 & 111.311 & & 96.481 & 0.0321 \\
China + South Korea & 3169.281 & 56.296 & 41.809 & 0.0141 \\
China + Japan & 31391.746 & 177.177 & 162.721 & 0.0546 \\
China + Japan + South Korea & 27797.351 & 166.725 & 149.155 & 0.0498
\end{tabular}

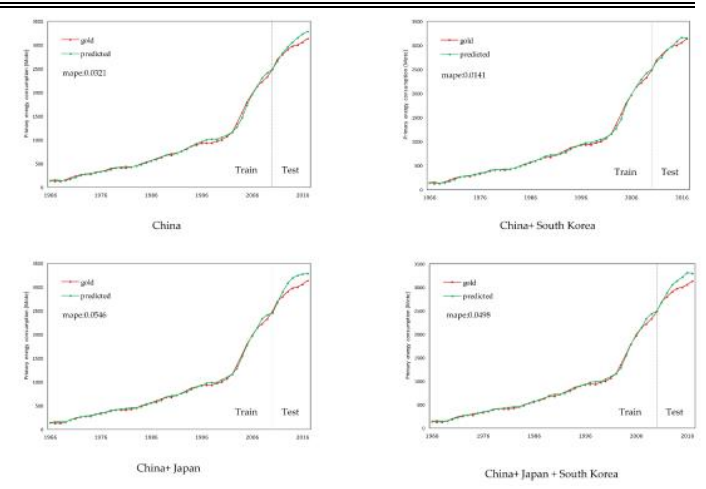

FIGURE 2. Comparison of the predicted and actual value of 


\section{China's primary energy consumption}

When Japan is the target country, four combinations, namely, Japan, Japan+China, Japan+South Korea, and Japan+China+South Korea, are used for the collaborative forecast based on SVR. As above, MSE, RMSE. MAE and MAPE are the error indicators. The errors of the four combinations for the prediction of the total annual primary energy consumptions of Japan are shown in Table 3. It can be seen that when using the combination of Japan+South Korea, the errors are the smallest. That is to say, when the primary energy consumption forecast of Japan implements the coordinated prediction of the two-source energy consumption portfolio of Japan + Korea, the improvement effect is very obvious. For the given time span (1965 to 2017), the comparison of predictive values and actual values of the total primary energy consumption of Japan by using the four combinations is shown in Fig. 3.

TABLE III.

Japan's primary energy consumption forecast is based on the comparison of results of primary energy composition in different countries

\begin{tabular}{cccccc}
\hline \hline Primary energy combination & MSE & RMSE & MAE & MAPE \\
\hline Japan & 1832.696 & 42.810 & 41.2978 & 0.0907 \\
\hline Japan + China & 10583.421 & 102.876 & 96.280 & 0.2119 \\
\hline Japan + South Korea & 284.222 & 16.859 & 14.168 & 0.0309 \\
\hline & 7474.473 & 86.455 & 75.837 & 0.1674 \\
\hline & & & & & \\
\hline
\end{tabular}

FIGURE 3.Comparison of the predicted and actual value of

\section{Japan's primary energy consumption}

when South Korea is the target country, four combinations, namely, South Korea, South Korea+China, South Korea+Japan, and South Korea+China+Japan, are used for the collaborative forecast based on SVR. As above, MSE, RMSE, MAE and MAPE are the error indicators. The errors of the four combinations for the prediction of South Korea's total annual primary energy consumption are shown in Table 4. It can be observed when using the combination of Japan+South Korea, the errors are the smallest. Therefore, the multi-source data collaboration approach does not achieve a much better effect in the case of South Korea. For the given time span (1965 to 2017), the comparison of the predictive and actual values of South Japan's total primary energy consumption using the four combinations is shown in Fig. 4.

$$
\text { Table IV. }
$$

South Korea's primary energy consumption forecast is based on the comparison of results of primary energy composition in different

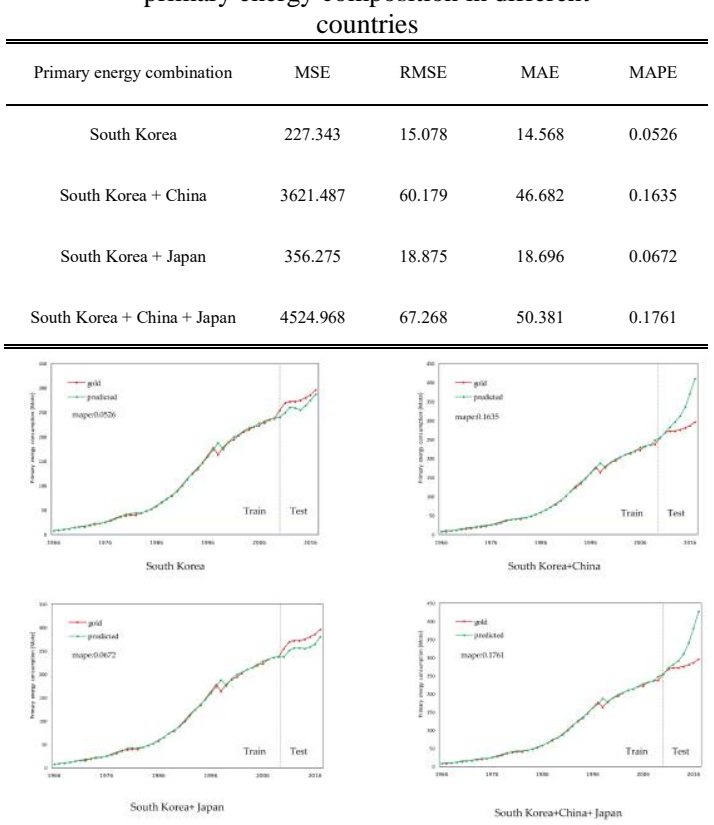

FIGURE 4. Comparison of the predicted and actual value of South Korea's primary energy consumption

\section{DISCUSSION}

It can be observed from the experiment that the prediction of China's primary energy consumption is reduced with the cooperation of South Korea. It is clear to see from Table 2 that the multi-source data collaborative prediction model can reduce the prediction error more effectively than the primary energy prediction of a single country. Similarly, in the forecast of Japan's total primary energy consumption, the combination of Japan's and South Korea's primary energy consumption can achieve the best forecast result (table 3). Finally, in the prediction of South Korea, when the primary energy composition of South Korea is only used in this paper to predict the total primary energy consumption, MAPE is the lowest (table 4), indicating that the primary energy consumption of South Korea is not affected by the primary energy consumption of China and Japan.

This finding can be explained by the energy imports and exports among the three countries. Take South Korea as an example, the fossil energy in South Korea is very scarce, and the 
primary energy consumption mainly includes oil, coal, natural gas and nuclear energy (figure 2 ), of which coal, oil and natural gas are mostly imported. But South Korea has a large and well-established petroleum industry, and petroleum products have long been its major export commodities. China and Japan are faced with shortage of petroleum but a huge demand for petroleum, and these two countries rely on the imports of petroleum[43]. As the neighboring country of China and Japan and also the major producer of petroleum products, South Korea is the exporter of petroleum products for China and Japan (Fig. 5). The change of South Korea's primary energy consumption influences the change of South Korea's oil export products. China and Japan import a lot of south Korean petroleum products, and the change of south Korean petroleum products affects the primary energy consumption of China and Japan.

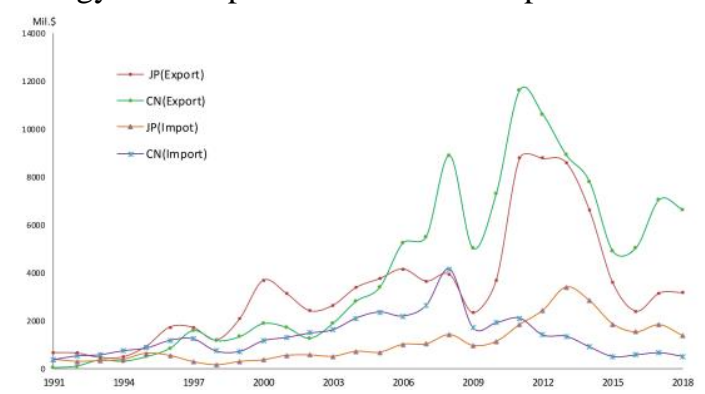

FIGURE 5. South Korea imports and exports oil products to

China and Japan

SVR model is built in this paper to study the correlations with primary energy consumption of China and Japan as continuous variables and oil products exported by South Korea to China and Japan as response variable. The primary energy consumptions of South Korea and China are directly proportional to the exports of petroleum products to China. (Fig. 6). In other words, South Korea's primary energy consumption influences that of China, the former plays an important role in China's primary energy forecast. (Table 2). In the structure of energy trade between China and South Korea, it is necessary to attach importance to the oil trade between China and South Korea, improve trade policies, increase the proportion of oil products imported by South Korea, and establish a china-south Korea oil product trade system with lower transportation costs.

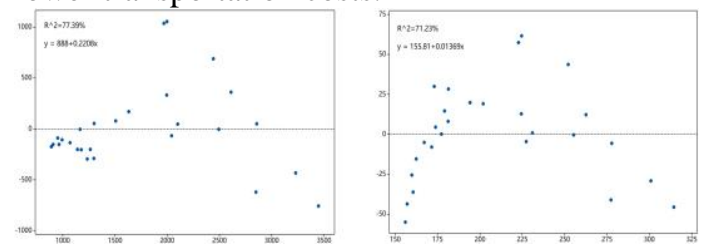

FIGURE 6. The residual chart of China and South Korea's primary energy consumption with output of petroleum products to China

For Japan, South Korea's primary energy consumption is directly proportional to the exports of petroleum products to Japan. Japan's primary energy consumption is inversely proportional to the exports of petroleum products to Japan. (Fig. 7). In other words, South Korea's primary energy consumption influences that of Japan, and the former has a strong predictive power on the latter (Table 3). For South Korea, 95\% of fossil energy is imported, and the main importers are the Middle East countries such as Saudi Arabia, Kuwait, the United Arab Emirates and Qatar. Therefore, primary energy consumptions of neighboring countries have little impact on that of South Korea, which explains the small predictive power of primary energy consumptions of China and Japan on that of South Korea (Table 4). In energy trade among the three countries, South Korea is the major exporter of petroleum products and hub of regional energy cooperation, as well as plays an important role in solving petroleum shortage of the three countries.
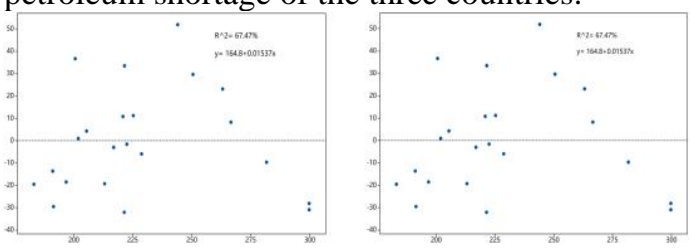

FIGURE 7. The residual chart of Japan and South Korea's

primary energy consumption with output of petroleum

products to Japan

In this paper, the prediction accuracy of primary energy consumption in China, Japan and South Korea is improved by using multi-source data for collaborative prediction, and the correlation between primary energy consumption is verified. Through the analysis, it is found that the energy consumption of South Korea influences the primary energy consumption of China and Japan, which is mainly related to the large amount of oil products exported by South Korea, and China and Japan are important importers. While maintaining sufficient oil reserves by imports from other countries, China and Japan will export excess oil to South Korea at a low price. South Korea, with its complete petroleum processing system, manufactures petroleum products in large quantities, which are then exported to China and Japan at a low price. This regional energy cooperation system, dominated by petroleum cooperation, can help lessen the shortage of fossil energy of the three countries. The research has demonstrated the correlations 
about primary energy consumptions of the three countries through accurate prediction of primary energy consumptions based on the multi-source data collaboration.

\section{VI.CONCLUSION}

China, Korea and Japan, three countries in East Asia, are selected as the research objects. The primary energy consumption of different countries is forecast collaboratively using different combinations of primary energy components by SVR. The analysis shows that South Korea's primary energy consumption has a major predictive power on that of Japan and China. We further propose recommendations for upgrading regional energy cooperation mode. By predicting the primary energy consumptions of the three countries, the following conclusions are drawn:

(1) Regional collaborative prediction model is built, and combinations of primary energy consumption composition data of different countries are used as the characteristic variables. Correlations between primary energy consumptions of neighboring countries are observed, which can be utilized to predict primary energy consumptions of single target country.

(2) Among all the experimental results, MAPE $<6 \%$ is the best prediction model for each country. For the prediction of China's primary energy consumption, the optimal prediction model has MAPE $=1.41 \%$, which indicates excellent performance of the prediction model.

(3) The accuracy of collaborative forecast is the highest for China when the combination of China and South Korea is used. The combination of Japan and South Korea shows the highest accuracy of collaborative forecast for the primary energy consumption of Japan. Moreover, the prediction accuracy of Korea's primary energy consumption is the highest by using Korea's primary energy consumption composition alone.

(4) Through the analysis, it is found that the impact of Korean energy consumption on primary energy consumption in China and Japan is mainly related to Korea's large export of petroleum products, and China and Japan are important importers. A new energy cooperation mode with Korea as the energy link should be established among the three countries to play the central role of Korea and upgrade energy cooperation pattern.

Research on regional collaborative forecast of primary energy consumptions based on different combinations of countries can be furthered in the following respects: First, it is important to identify which energy consumption contributes most to the prediction in different primary energy consumption components in different countries. Second, deep learning model can be introduced in order to discard the choice of different energy consumption among different countries. That way, the model will automatically extract valuable information for the forecast. Third, multi-source data collaboration approach can be extended to a larger scope in the Asia-Pacific region, and the correlations between primary energy consumptions of other countries can be studied in the future.

\section{ACKNOWLEDGMENT}

This work was supported in part by the National Natural Science Foundation of China under Grant 71503180 and Humanities And Social Science Fund Of Ministry Of Education Of China under grant number 20YJA630042

\section{Author Contributions}

Bingchun Liu conceived and designed the experiments; Peng Zhang performed the experiments; both together wrote the paper and field edited the paper; Qingshan Wang contributed analysis tools and oversaw the manuscript's content and production.

\section{Conflicts of Interest}

The authors declare no conflict of interest.

\section{REFERENCES}

[1] Qiang, Ji.; Zhang, H.Y.; Fan, Y. Identification of global oil trade patterns: An empirical research based on complex network theory. Energy Convers Manage. $2014,85,856-865$.

[2] An ,H.Z.; Zhong, W.Q.; Chen ,Y.R.; Li ,H.J.; Gao X.Y. Features and evolution of international crude oil 
trade relationships: A trading-based network analysis. Energy. 2014,74,254-259.

[3] Zhong, W.Q.; An, H.Z.; Gao, X.Y.; Sun, X.Q. The evolution of communities in the international oil trade network. Physica A. 2014,413,42-52.

[4] Guan, Q.; An, H.Z., Hao, X.Q., Jia, X.L. The impact of countries' roles on the international photovoltaic trade pattern: the complex networks analysis. Sustain-Basel. 2016;8.

[5] BP Statistical Review of World Energy. Available online:https://www.bp.com/content/dam/bp/en/corpora te/pdf/energy-economics/statistical-review/bp-stats-re view-2018-full-report.pdf(accessed on June 2018).

[6] Wu, X.F.; Chen, G.Q. Global primary energy use associated with production, consumption and international trade.Energy Policy.2017,111,85-94.

[7] Gozgor, G. Energy consumption and economic growth: New evidence from the OECD countries.Energy.2018,153,27-34.

[8] Chen, T. Analyzing and forecasting the global $\mathrm{CO} 2$ concentration-a collaborative fuzzy-neural agent network approach. Journal of Applied Research and Technology.2015,13,364-373.

[9] Li, M.L. Forecasting Carbon Emissions Related to Energy Consumption in Beijing-Tianjin-Hebei Region Based on Grey Prediction Theory and Extreme Learning Machine Optimized by Support Vector Machine Algorithm. Energies. 2018,11,2475.

[10] Wu, X.; Kumar, V. The Top Ten Algorithms in Data Mining; Taylor \& Francis Group: New York, NK, USA, 2009.

[11] Xiao, J. A hybrid model based on selective ensemble for energy consumption forecasting in China.Energy.2018,159,534-546.

[12] Wang, Q. Forecasting Energy Demand in China and India: Using Single-linear, Hybrid-linear, and Non-linear Time Series Forecast Techniques.Energy.2018,161,821-831.

[13] Brinda, Mahalingam. GDP and energy consumption: A panel analysis of the USA. Applied Energy.2018,213,208-218

[14] Luisa F. Cabeza. Renewable energy research and technologies through responsible research and innovation looking glass: Reflexions, theoretical approaches and contemporary discourses. Applied Energy.2018,211,792-808.

[15] Chen, G.Q.; Han, M.Y. Global supply chain of arable land use: production-based and consumption-based trade imbalance. Land Use Policy.2015,49,118-130.

[16] Vincenzo Bianco. Long term outlook of primary energy consumption of the Italian thermoelectric sector: Impact of fuel and carbon prices.Energy.2015,87,153-164.

[17] Yuan, C.Q. Comparison of China's primary energy consumption forecasting by using ARIMA (the autoregressive integrated moving average) model and GM(1,1) model. Energy.2016,100,384-390.

[18] Li, J.R.; Wang, R.; Wang, J.Z.; Li Y.F. Analysis and forecasting of the oil consumption in China based on combination models optimized by artificial intelligence algorithms. Energy. 2018,44,43-64.

[19] Sasan, Barak.Forecasting energy consumption using ensemble ARIMA-ANFIS hybrid algorithm. International Journal of Electrical Power \& Energy Systems.2016,82,92-104.

[20] T. Chen. An effective fuzzy collaborative forecasting approach for predicting the job cycle time in wafer fabrication. Computers \& Industrial Engineering.2013,66,834-848.

[21] Can Eksoz. Collaborative forecasting in the food supply chain: A conceptual framework. International Journal of Production Economics.2014,158,120-135.

[22] T. Chen. Analyzing and forecasting the global $\mathrm{CO} 2$ concentration-a collaborative fuzzy-neural agent network approach. Journal of Applied Research and Technology.2015,13,364-373.

[23] Sayyed Mahdi Ziaei. Effects of financial development indicators on energy consumption and $\mathrm{CO} 2$ emission of European, East Asian and Oceania countries.Renewable and Sustainable Energy Reviews.2015,42,752-759.

[24] UsamaAl-mulali. Econometric analysis of trade, exports, imports, energy consumption and $\mathrm{CO} 2$ emission in six regions. Renewable and Sustainable Energy Reviews.2014,33,484-498.

[25] Giray Gozgor. Energy consumption and economic growth: New evidence from the OECD countries.Energy.2018,153,27-34. 
[26] Topcu.T. Further evidence on the trade-energy consumption nexus in OECD countries. Energy Policy.2018,117,160-165.

[27] Alex J. Smola. The connection between regularization operators and support vector kernels. Neural Networks.1998,11,637-649.

[28] Andrés García-Floriano. Support vector regression for predicting software enhancement effort. Information and Software Technology.2018,97,99-109.

[29] Yang, W.T. Prediction of hourly PM2.5 using a space-time support vector regression model. Atmospheric Environment.2018,181,12-19.

[30] Petra Vrablecová. Smart grid load forecasting using online support vector regression. Computers \& Electrical Engineering.2018,65,102-117.

[31] Gelayol Golkarnarenji. Support vector regression modelling and optimizationof energy consumption in carbon fiber production line.2018.109,276-288.

[32] Yang, Y.L. An incremental electric load forecasting model based on support vector regression.Energy.2016,113,796-808.

[33] Chen, Y.B. Short-term electrical load forecasting using the Support Vector Regression (SVR) model to calculate the demand response baseline for office buildings. Applied Energy.2017,195,659-670.

[34] Abdolreza Nazemi. Improving corporate bond recovery rate prediction usingmulti-factor support vector regressions .European Journal of Operational Research.2018,271,664-675.

[35] A.Khosravi. Time-series prediction of wind speed using machine learning algorithms: A case study
Osorio wind farm, Brazil. Applied Energy.2018,224,550-566.

[36] Vapnik V, Golowich S, Smola A. Support vector method for function approximation, regression estimation, and signal processing. Advances in Neural Information Processing Systems. 1997; 9,281-287.

[37] Federico Scarpa. Assessing the Quality of Natural Gas Consumption Forecasting: An Application to the Italian Residential Sector . Energies . 2017, 10, 1879.

[38] Liu, Y.L; Wu, Z; Fu, H.X. Scenario Analysis of Natural Gas Consumption in China Based on Wavelet Neural Network Optimized by Particle Swarm Optimization Algorithm. Energies .2018, 11, 825.

[39] Torrini, F.C.; Souza, R.C.; Oliveira, F.L.C.; Pessanha, J.F.M. Long term electricity consumption forecast in Brazil: A fuzzy logic approach. Socio-Econ. Plan. Sci. 2016, 54, 18-27.

[40] Gokhan, A. Modeling of energy consumption based on economic and demographic factors: The case of Turkey with projections. Renew. Sustain. Energy Rev. $2015,35,382-389$.

[41] Shaikh, F.; Ji, Q.; Shaikh, P.H.; Mirjat, N.H.; Uqaili, M.A. Forecasting China's natural gas demand based on optimised nonlinear grey models. Energy. 2017 , $140,941-951$.

[42] Akpinar, M.; Yumusak, N. Year ahead demand forecast of city natural gas using seasonal time series methods. Energies. 2016, 9, 727. 


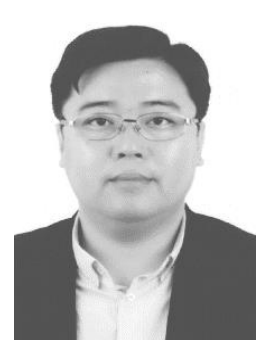

SECOND

\section{A. AUTHOR}

Bingchun Liu was born in

Hengshui City, Hebei Province,

China in 1977. He received the B.S.

degrees in business administration

from Hebei University of

Technology, Tianjin, China, in 2002

and received the M.S. degrees in management science and engineering from Hebei University of Technology, Tianjin,

China, in 2005 and the Ph.D. degree in technological economics and management from Tianjin University, Tianjin,

China, in 2011.

From 2012 to 2016, he worked in the Post-doctoral Mobile Station of Management Science and Engineering of Hebei University of Technology. Since 2014, he has been an Associate Professor with Management Department of Tianjin University of Technology. He is the author of three books and more than 30 articles. His research interest includes regional economy and industrial ecology, big data and deep learning algorithm optimization, logistics system planning and design and transportation economics.

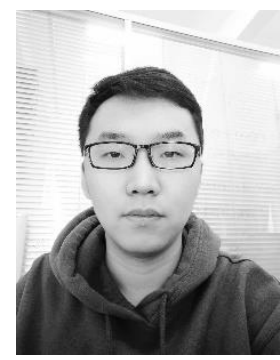

SECOND B. AUTHOR Peng

Zhang received the B.S. degree in logistics management from Tianjin University of Technology, Tianjin, China, in 2017. He is currently pursuing for M.S. degree in logistics management in Tianjin University of technology, Tianjin, China. His research interest includes big data and deep learning algorithm optimization and regional energy system optimization.

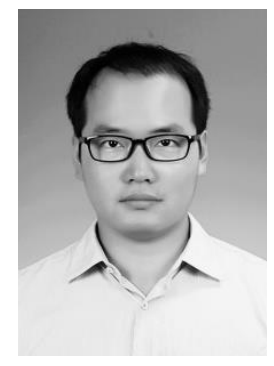

\section{SECOND C. AUTHOR}

Qingshan Wang received the

B.S. degrees in mathematics and applied mathematics from Tianjin University of Technology, Tianjin, China, in 2010 and received the

M.S. degrees in Management and science from Tianjin University of Technology, Tianjin, China, in 2013 and the Ph.D. degree in business administration from Tianjin University, Tianjin, China, in 2016.

Since 2016, he has been a Lecturer with Humanities Department of Tianjin Agricultural University. His research interest includes regional economy and industrial ecology, big data and deep learning algorithm optimization. 